Article

\title{
Pathway Analysis and Metabolites Identification by Metabolomics of Etiolation Substrate from Fresh-Cut Chinese Water Chestnut (Eleocharis tuberosa)
}

\author{
Yi-Xiao Li, Yong-Gui Pan *, Feng-Ping He, Meng-Qi Yuan and Shang-Bin Li \\ College of Food, Hainan University, Haikou 570228, China; lisa19910@163.com (Y.-X.L.); \\ hfping123@126.com (F.-P.H.); sullna@163.com (M.-Q.Y.); sharplee1208@163.com (S.-B.L.) \\ * Correspondence: yongui123@126.com; Tel.: +86-898-66156125
}

Academic Editors: Pinarosa Avato, Baodong Zheng and Jianbo Xiao

Received: 9 October 2016; Accepted: 21 November 2016; Published: 1 December 2016

\begin{abstract}
Fresh-cut Chinese water chestnuts (CWC) turn yellow after being peeled, reducing their shelf life and commercial value. Metabolomics, the systematic study of the full complement of small molecular metabolites, was useful for clarifying the mechanism of fresh-cut CWC etiolation and developing methods to inhibit yellowing. In this study, metabolic alterations associated with etiolation at different growth stages ( 0 day, 2 days, 3 days, 4 days, 5 days) from fresh-cut CWC were investigated using LC-MS and analyzed by pattern recognition methods (principal component analysis (PCA), partial least squares-discriminant analysis (PLS-DA), and orthogonal projection to latent structures-discriminant analysis (OPLS-DA)). The metabolic pathways of the etiolation molecules were elucidated. The main metabolic pathway appears to be the conversion of phenylalanine to $p$-coumaroyl-CoA, followed by conversion to naringenin chalcone, to naringenin, and naringenin then following different pathways. Firstly, it can transform into apigenin and its derivatives; secondly, it can produce eriodictyol and its derivatives; and thirdly it can produce dihydrokaempferol, quercetin, and myricetin. The eriodictyol can be further transformed to luteolin, cyanidin, dihydroquercetin, dihydrotricetin, and others. This is the first reported use of metabolomics to study the metabolic pathways of the etiolation of fresh-cut CWC.
\end{abstract}

Keywords: Chinese water-chestnut; metabolomics; LC-MS; metabolites; pathway analysis

\section{Introduction}

Metabolomics, a new field in systems biology, represents an emerging and powerful discipline concerned with the comprehensive analysis of small molecules $(<1 \mathrm{kDa})$; it provides a powerful approach for the discovery of biomarkers in biological systems [1-4]. Metabolomics provides a quantitative analysis of all low-molecular-mass metabolites and lends insight into the relationship between metabolites and changes in physiology/pathophysiology status in biochemical networks and pathways by combining a range of different analytical technologies and calculation methods [5-7]. These techniques have recently demonstrated significant potential in many fields, such as toxicology [8], nutrition [9], environmental stress [10], global effects of genetic manipulation, disease diagnosis, drug mechanism and development, natural product discovery, and comparison of different growth stages [11,12]. Additionally, metabolomics has been used in food science to assess food quality, food safety, food microbiology, and food processing [13-15]. Furthermore, the global metabolite profile now enables much more detailed studies of noninvasive biomarkers that can detect different growth stages in plants.

Plant studies using metabolomics techniques aim for the simultaneous detection of all metabolites in plant tissues. Metabolomics detection technology includes nuclear magnetic resonance (NMR) 
spectroscopy, liquid chromatography-mass spectrometry (LC-MS) [16], and gas chromatography-mass spectrometry (GC-MS). Among these, GC-MS is mainly suited for compound classes appearing mainly in primary metabolic pathways, such as amino acids, fatty acids, carbohydrates, and organic acids [17]. LC-MS is more suitable for assessing the overall biochemical richness of plants. This technique analytically covers the large (semipolar) group of plant secondary metabolites including alkaloids, saponins, phenolic acids, phenylpropanoids, flavonoids, glucosinolates, polyamines, and their derivatives $[18,19]$. Therefore, LC-MS is preferred over the other techniques in the study of numerous structure databases [20]. At present, LC-MS has become one of the frequently used techniques in metabolomics studies because of its high sensitivity and reproducibility.

The Chinese water chestnut (CWC, Eleocharis tuberosa) is one of the most popular hydrophytic vegetables in China because of its unique taste [21]. However, it is usually peeled before being eaten, and fresh-cut CWC turns yellow after being peeled, reducing its shelf life and its commercial value. Recent studies have shown that CWC etiolation is linked to the presence of eriodictyol and naringenin [21]. In addition, most researches have shown that phenylalanine ammonia-lyase (PAL) was present in fresh-cut CWC tissue. With the extension of storage time, the activity of PAL increases and the etiolation of CWC also increases. Therefore, the metabolic pathways of eriodictyol and naringenin are believed to be associated with PAL activity [22]. PAL is a critical and rate-limiting enzyme in the phenylpropanoid metabolic pathway, a pathway that also contains eriodictyol and naringenin [23-26]. It is therefore probable that the etiolation process of fresh-cut CWC is related to the functioning of the phenylpropanoid metabolic pathway. However, there may be a variety of specific metabolic pathways related to yellowing, and few prior studies have investigated these pathways fully.

The synthesis of flavonoids begins with the shikimic acid metabolic pathway, which generates phenylalanine. This molecule then enters the phenylpropanoid metabolic pathway, and then, through the common enzyme chalcone synthase, enters the flavonoid synthesis pathway, which generates the various flavonoids through different branches $[27,28]$. Though the use of metabolomics to research flavonoids is relatively uncommon, both naringenin and eriodictyol are considered flavonoids, and the molecular weight of most flavonoids is below $1 \mathrm{kDa}$; therefore, the metabolites of the etiolation process are suitable for studying with metabolomics techniques. Literature about the process of involving metabolomics in the research of flavonoids is very common, especially in herbal aspects [29,30]. In terms of fruit and vegetables, the glycosides of luteolin and apigenin as well as quercetin conjugates were identified from Phoenix dactylifera (date palm) fruits using UPLC/PDA/ESI-TOF-MS methods [31,32]. Flavonoids in blueberry extracts were analyzed through HPLC-ESI-MS/MS, which identified that anthocyanins and flavonoid alcohols are the main components [32]. Therefore, there is precedence for identifying the characteristic flavonoids and metabolic pathways from plants.

In order to clarify the mechanism of fresh-cut CWC etiolation and yellowing, as well as to evaluate the safety of the etiolation metabolite, we sought to discover the metabolic pathways of the molecules responsible for etiolation. In this study, we used the LC-MS method combined with additional analytical technologies and calculation methods (principal component analysis (PCA), partial least squares-discriminant analysis (PLS-DA), orthogonal projection to latent structures-discriminant analysis (OPLS-DA)) to analyze the metabolic pathways of etiolation in fresh-cut CWC.

\section{Results and Discussion}

\subsection{LC-MS Analysis of Metabolic Patterns}

In this study, five time points from the yellowing process of CWC were studied using an LC-MS metabolomics approach. For the analysis, we compared 0 day (see Methods) with 2 days (0-2), 2 days with 3 days (2-3), 3 days with 4 days (3-4), and 4 days with 5 days (4-5) for screening and identification of the metabolites. According to peak height data, we detected 1046 positive-ion (ESI+) peaks and 1962 negative-ion (ESI-) peaks by Mass Profiler using the same acquiring method of 0.5-15 min of retention time, and then the normalized data were fed to SIMCA-P. The parameters $\left(R^{2} X, R^{2} Y\right.$, and 
$Q^{2} Y$ ) obtained from SIMCA-P indicated that the model was able to distinguish between each of the yellowing time points in both ion modes.

To further evaluate the capability of the LC-MS-based metabolomics approach to distinguish between all of the time points, multivariate data analyses (PCA, PLS-DA, OPLS-DA) were performed. In the PCA analysis, we identified five principal components in positive mode $\left(R^{2} X=0.464, Q^{2}=0.124\right)$ (Figure 1). For this nonsupervisory PCA model, the $\mathrm{R}^{2} \mathrm{X}$ parameter-which describes the rate of the model-can be used to determine the model's quality. Generally, a model is reliable when $R^{2} X>0.4$ [33]. Thus, the current PCA model was suited to explain the metabolic differences between each group. Every point in Figure 2 represents a sample with the fresh-cut CWC; from the PCA analysis it can be visually seen that there is a significant difference between each pair of time points, except for between 3 days and 4 days. Furthermore, the between-group differences were significantly greater than the intergroup differences, indicating that substantial biochemical perturbation occurred in all groups. PCA as an unsupervised analysis method, responsive to the primitive state of the data, can be used to observe the relationship between each group, but it cannot account for intergroup errors or eliminate the random error unrelated to this research. In order to obtain information on which metabolites were the cause of the differences, we conducted PLS-DA and OPLS-DA analyses for each pair of time points.

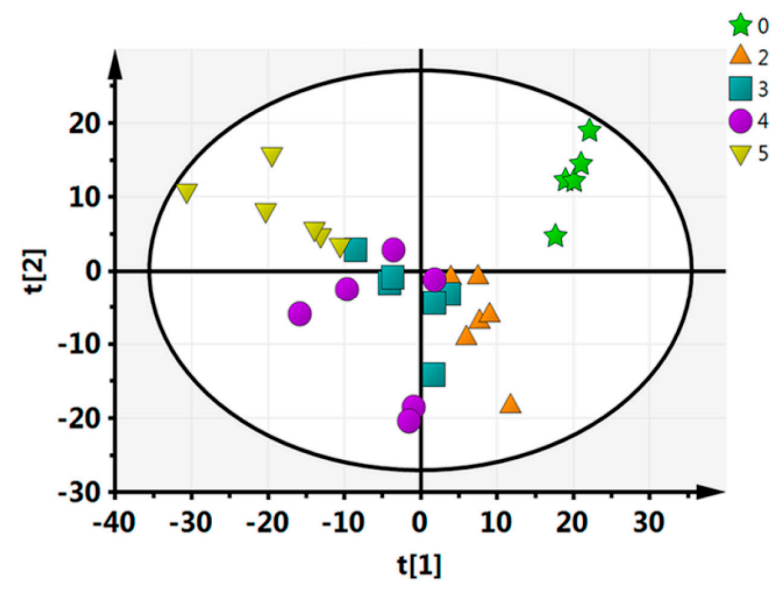

Figure 1. Statistical principal component analysis (PCA) analysis of the 5 time points.

The supervised PLS-DA analysis revealed greater differences in the metabolic signature between each pair of time points, focusing on intergroup differences and minimizing between-group differences to better grasp the overall characteristics and variation of the data. For each pair of time points studied, this analysis was performed in both the positive mode and negative mode. Results for each pair are as follows (Figure 2): in positive mode, groups $0-2$ obtained one principal component $\left(R^{2} X=0.253\right.$, $\left.R^{2} Y=0.97, Q^{2}=0.845\right)$; groups $2-3$, one principal component was obtained $\left(R^{2} X=0.328, R^{2} Y=0.923\right.$, $\left.\mathrm{Q}^{2}=0.68\right)$; groups 3-4 obtained one principal component $\left(\mathrm{R}^{2} \mathrm{X}=0.179, \mathrm{R}^{2} \mathrm{Y}=0.897, \mathrm{Q}^{2}=0.588\right)$; groups 4-5, one principal component was obtained $\left(R^{2} X=0.198, R^{2} Y=0.867, Q^{2}=0.514\right)$; in negative mode, groups 0-2 obtained one principal component $\left(R^{2} X=0.278, R^{2} Y=0.945, Q^{2}=0.829\right) ; 2-3$, one principal component was obtained $\left(R^{2} X=0.298, R^{2} Y=0.913, Q^{2}=0.787\right)$; groups 3-4 obtained two principal components $\left(R^{2} X=0.321, R^{2} Y=0.991, Q^{2}=0.806\right)$; groups $4-5$, one principal component was obtained $\left(R^{2} X=0.259, R^{2} Y=0.879, Q^{2}=0.712\right)$. The model parameters $\left(R^{2} X\right.$ and $\left.Q^{2}\right)$ indicate that the current PLS-DA models were reliable and suitable for interpretation between each pair of time points of metabolic differences and finding the differences. Meanwhile, the left of the arrange experiments were randomly generated depending on whether the value of $R^{2}$ and $Q^{2}$ was less than the original value from the right. This indicates that the predictive power of the original model is greater than any randomized $y$ variables, thus, the model is effective and it can continue to be applied to the different metabolites. 


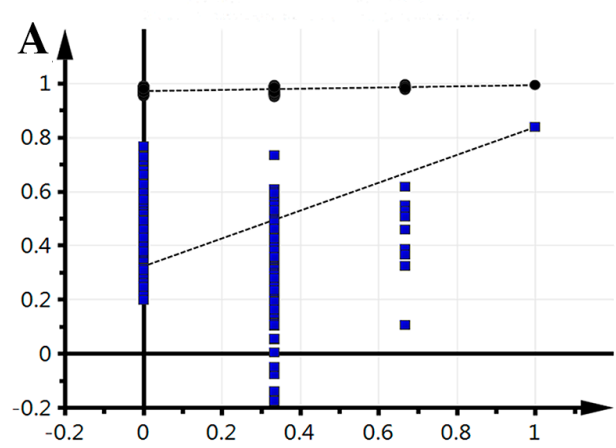

- R2

- Q2
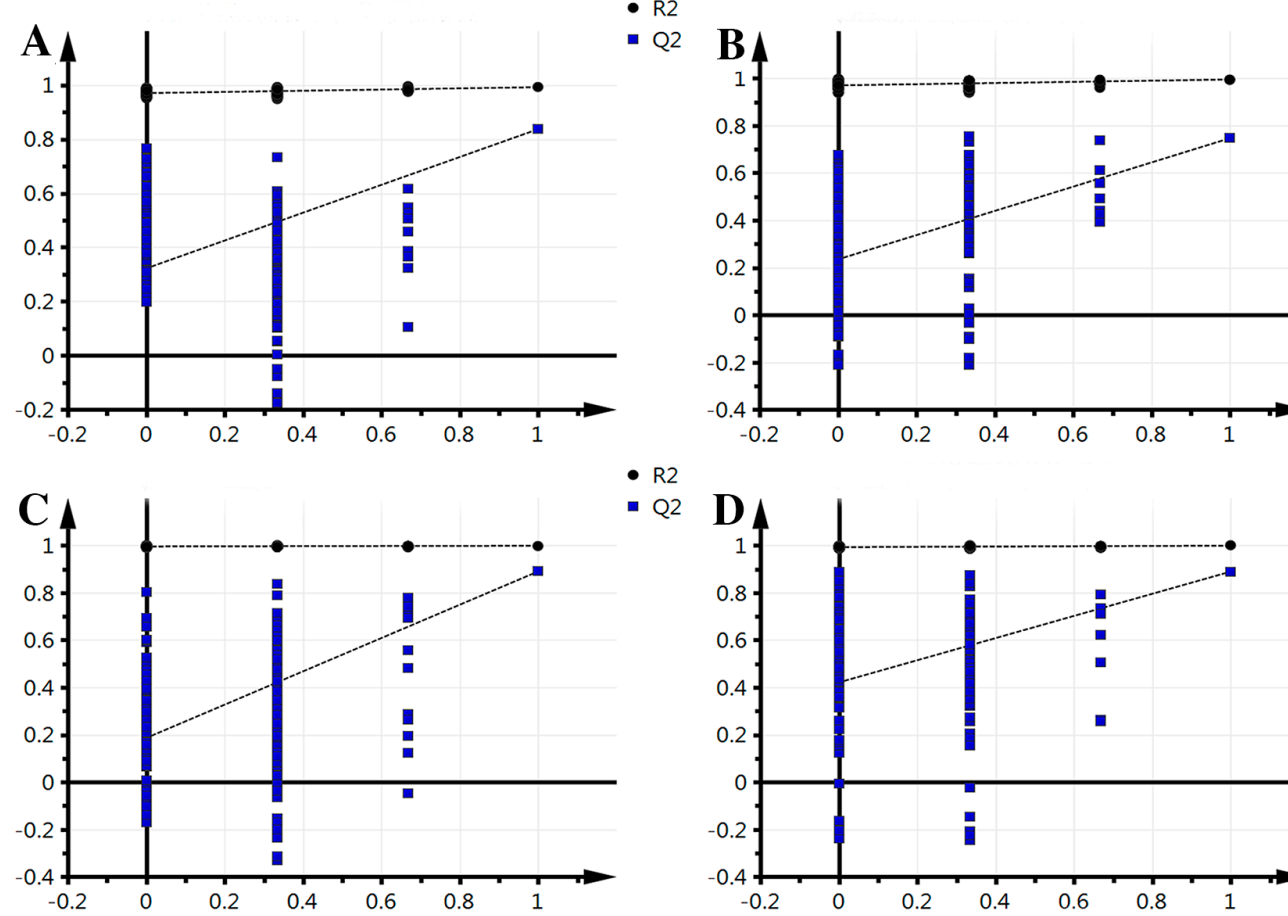

R2
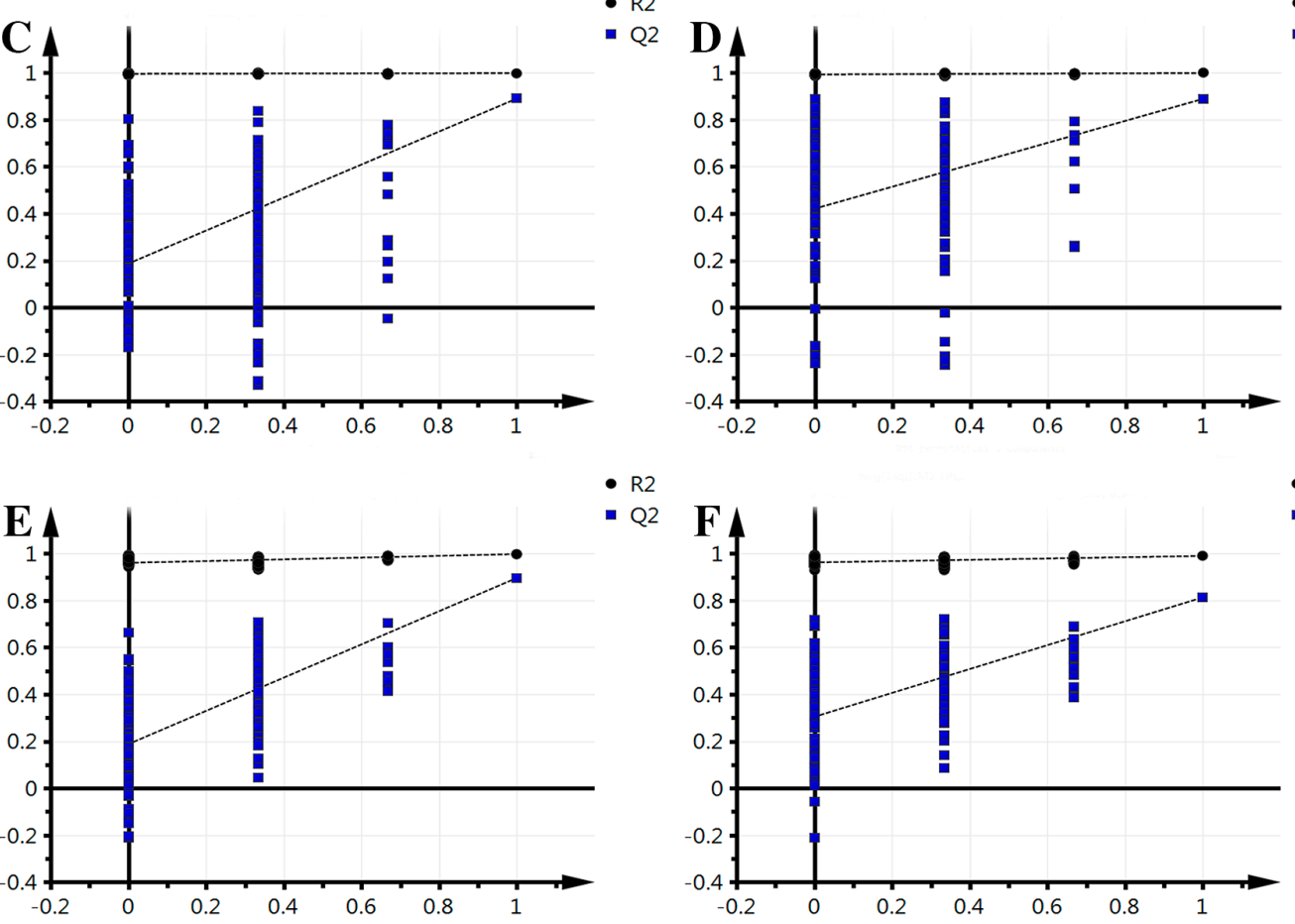

R2

Q2
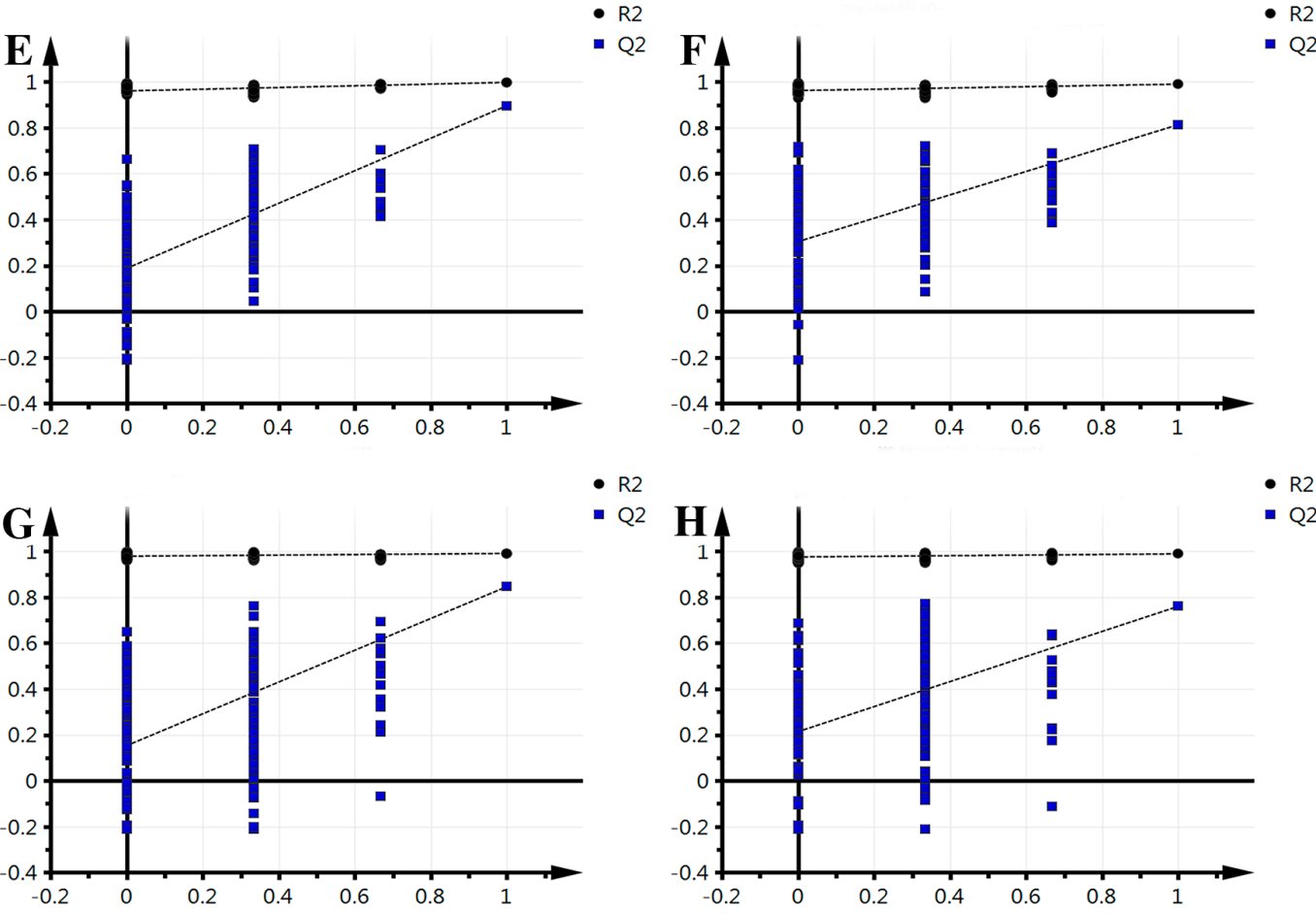

- R2

- Q2

R2

Q2

Figure 2. Effective verification of fresh-cut Chinese water chestnut during different storage times. (A) 0-2 comparison, ESI+; (B) 2-3 comparison, ESI+; (C) 3-4 comparison, ESI+; (D) 4-5 comparison, ESI+; (E) 0-2 comparison ESI-; (F) 2-3 comparison, ESI-; (G) 3-4 comparison, ESI-; (H) 4-5 comparison, ESI-.

Typically, OPLS-DA analysis is conducted to search for the different components that lead to the maximum differences between two samples. In order to further obtain the metabolic differences, this study applied an OPLS-DA approach; separating samples into two blocks. As can be seen from Figure 3, compared with PCA analysis, each group received the highest degree of separation, which is useful to determine the metabolic differences. 

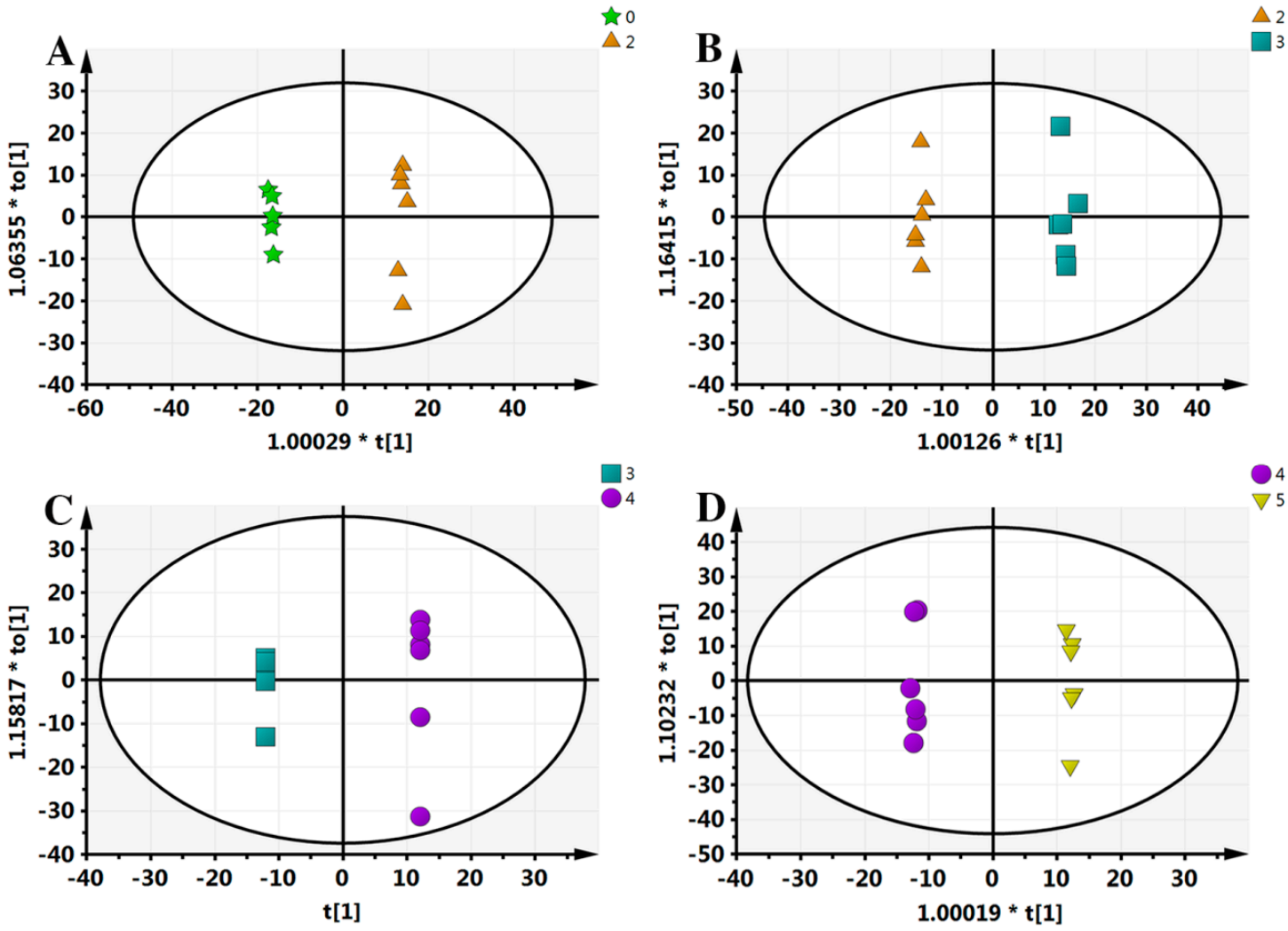

Figure 3. Orthogonal projection to latent structures-discriminant analysis (OPLS-DA) results of all samples. (A) 0-2 comparison, scanned by ESI+; (B) 2-3 comparison, ESI+; (C) 3-4 comparison, ESI+; (D) 4-5 comparison, ESI+. " "*" means the sample separation significantly.

\subsection{Identification of Metabolites}

All metabolites from each total ion chromatogram were extracted and aligned using Mass Profiler software. All information, including retention time, peak intensity, and exact mass, was supplied by the LC-Q/TOF-MS platform. The metabolites (VIP (variable importance in the projection) $>1$ ) were analyzed by $t$-test, and metabolites with a $p$-value $<0.05$ were identified as metabolic differences.

\subsubsection{Identification of Metabolites in 0-2 Days}

Comparing the samples of 0 day with 2 days, the main metabolite differences are shown in Tables 1 and 2. In the positive mode, $5^{\prime}$-deoxy-5'-(methylthio) adenosine, ferulic acid, and linolenic acid showed significant increases, while $\mathrm{N}$-palmitoyl threonine and stearidonic acid decreased. Among these, the linolenic acid at 2 days compared with 0 day increased by about 19 times. In the negative mode, salicylaldehyde, salicylic acid, naringenin, eriodictyol, pentahydroxyflavanone, apigenin-glucuronide, and luteolin 5-methyl ether 7-glucoside were increased significantly. The naringenin has increased by about 4820 times and the eriodictyol was increased by about 700 times, while the PE (phosphatidyl ethanolamine) (18:3), PE (18:1), and quercetin 3-O-(6"-malonyl-glucoside) 7-O-glucoside at 2 days compared with 0 day were decreased by 600 times. Thus, the metabolites between 0 day and 2 days have significant differences, and these metabolites were related to the pathways of fresh-cut CWC yellowing. 
Table 1. The main identification obtained between 0 day and 2 days in ESI+ $(p<0.05)$.

\begin{tabular}{cccccc}
\hline VIP & RT (min) & Mass & Name & T-Test & Fold (2/0) $^{*}$ \\
\hline 1.248 & 13.14 & 278.2252 & Linolenic Acid & 0.001 & +6.567 \\
1.408 & 4.91 & 194.0582 & ferulic acid & 0.000 & +4.237 \\
1.779 & 4.06 & 297.09 & 5'-Deoxy-5'-(methylthio)adenosine $^{\prime}$ & 0.001 & +4.040 \\
1.479 & 6.60 & 548.1175 & Chrysoeriol 7-O-(6"-malonyl-glucoside) & 0.000 & +2.683 \\
1.204 & 10.19 & 357.2886 & N-palmitoyl threonine & 0.009 & -2.457 \\
1.843 & 8.06 & 276.2095 & Stearidonic Acid & 0.006 & -2.086 \\
1.137 & 4.33 & 137.0477 & Anthranilic acid & 0.000 & -1.716 \\
1.181 & 4.39 & 264.137 & Abscisic Acid & 0.038 & -1.408 \\
1.668 & 0.82 & 115.064 & Proline & 0.007 & +0.275 \\
\hline
\end{tabular}

* 0 day and 2 days is the logarithm of the ratio of the value of value (base 2), " + " means 2 days increase relative to 0 day, "-" means decrease. VIP: Variable Importance in the Projection; RT: retention time.

Table 2. The main identification obtained between 0 day and 2 days in ESI- $(p<0.05)$.

\begin{tabular}{|c|c|c|c|c|c|}
\hline VIP & RT (min) & Mass & Name & $T$-Test & Fold $(2 / 0) *$ \\
\hline 1.612 & 7.57 & 272.0694 & Naringenin & 0.001 & +28.844 \\
\hline 1.778 & 5.82 & 288.0642 & Eriodictyol & 0.000 & +26.056 \\
\hline 1.773 & 6.11 & 462.1179 & Luteolin 5-methyl ether 7-glucoside & 0.000 & +24.638 \\
\hline 1.325 & 11.24 & 479.3027 & PE (18:1) & 0.017 & -24.315 \\
\hline 1.759 & 13.13 & 278.2251 & Linolenic Acid & 0.000 & 24.309 \\
\hline 1.153 & 3.47 & 712.1513 & Quercetin 3-O-(6"-malonyl-glucoside) 7-O-glucoside & 0.046 & -24.296 \\
\hline 1.624 & 4.87 & 304.0593 & Pentahydroxyflavanone & 0.001 & +24.082 \\
\hline 1.459 & 4.97 & 450.1182 & Eriodictyol 5-O-glucoside & 0.006 & +23.416 \\
\hline 1.398 & 10.03 & 475.271 & PE (18:3) & 0.010 & -23.115 \\
\hline 1.482 & 6.57 & 446.0862 & Apigenin-glucuronide & 0.005 & +22.622 \\
\hline 1.459 & 10.68 & 477.2872 & PE (18:2) & 0.006 & -5.758 \\
\hline 1.478 & 1.31 & 180.064 & Glucose & 0.005 & -3.408 \\
\hline 1.698 & 5.34 & 448.1027 & Naringenin- $4^{\prime}-O-\beta$-D-Glucuronide & 0.000 & +3.061 \\
\hline 1.339 & 5.21 & 670.1768 & Limocitrin 3,7-diglucoside & 0.015 & +2.829 \\
\hline 1.480 & 4.48 & 176.0687 & 2-Isopropylmaleate & 0.005 & +2.556 \\
\hline 1.245 & 1.11 & 668.1634 & Euphorbianin & 0.027 & +2.481 \\
\hline 1.634 & 4.91 & 180.0438 & Caffeic Acid & 0.001 & +2.355 \\
\hline 1.235 & 5.79 & 490.1128 & Cyanidin 3-(6-acetylgalactoside) & 0.029 & +2.216 \\
\hline 1.716 & 4.58 & 192.0629 & Quinic acid & 0.000 & +1.921 \\
\hline 1.515 & 5.58 & 207.0886 & $N$-Acetyl-L-phenylalanine & 0.003 & -1.786 \\
\hline 1.587 & 4.95 & 354.0962 & Chlorogenic Acid & 0.002 & 1.717 \\
\hline 1.608 & 8.12 & 492.1279 & Malvidin 3-O-glucoside & 0.001 & -0.310 \\
\hline 1.369 & 5.69 & 168.042 & Vanillic acid & 0.012 & -0.247 \\
\hline 1.369 & 6.30 & 272.0687 & Naringeninchalcone & 0.012 & -0.247 \\
\hline 1.369 & 9.73 & 356.1271 & Laurifolin & 0.012 & -0.247 \\
\hline 1.369 & 5.78 & 534.1009 & Cyanidin 3-O-(6-O-malonyl- $\beta$-D-glucoside) & 0.012 & -0.247 \\
\hline 1.369 & 5.23 & 535.1341 & Malvidin 3-(6-acetylglucoside) & 0.012 & -0.247 \\
\hline 1.369 & 6.31 & 558.1173 & Dihydromorelloflavone & 0.012 & -0.247 \\
\hline 1.369 & 6.97 & 754.1943 & Luteolin 7-glucuronide- $3^{\prime}, 4^{\prime}$-dirhamnoside & 0.012 & -0.247 \\
\hline 1.369 & 8.24 & 784.1795 & Apigenin 7-Glucosyl-(1->2)-glucuronide-4'-glucuronide & 0.012 & -0.247 \\
\hline
\end{tabular}

$* 0$ day and 2 days is the logarithm of the ratio of the value of value (base 2$),$ " + " means 2 days increase relative to 0 day, "-" means decrease. VIP: Variable Importance in the Projection; RT: retention time.

\subsubsection{Identification of Metabolites in 2-3 Days}

Comparing the samples of 2 days with 3 days, the main metabolite differences are shown in Tables 3 and 4 . In the positive mode, cyanidin 3-O-(6-O-malonyl- $\beta$-D-glucoside) and dihydroxy$6,7,4^{\prime}, 5^{\prime}$-tetramethoxyflavone increased significantly, while aconitic acid and MG (18:2) decreased. In the negative mode, phenylacetic acid, vanillic acid, quinic acid, quercetin and its derivatives, dihydroxy-dimethoxy-methylenedioxy, the derivatives of naringenin, malvidin, and luteolin were increased significantly, all of them showing an average increase of about 280 times. In contrast, ascorbic acid and arginine at 3 days, compared with 2 days, were decreased by 4 times. The major differences were identified to further explore the pathway of CWC yellowing. 
Table 3. The main identification obtained between 2 days and 3 days in ESI+ $(p<0.05)$.

\begin{tabular}{cccccc}
\hline VIP & RT (min) & Mass & Name & T-Test & Fold (3/2) \\
\hline 1.482 & 10.60 & 354.2777 & MG (18:2) & 0.023 & -3.108 \\
1.333 & 6.11 & 534.1018 & Cyanidin 3-O-(6-O-malonyl- $\beta$-D-glucoside) & 0.010 & +2.596 \\
1.081 & 1.04 & 174.0166 & Aconitic acid & 0.007 & -2.281 \\
1.037 & 5.95 & 374.0975 & Dihydroxy-6,7, $4^{\prime}, 5^{\prime}$-tetramethoxyflavone & 0.022 & +2.078 \\
1.656 & 6.34 & 316.0954 & Dihydroxy-dimethoxyflavanone & 0.018 & +0.879 \\
1.449 & 0.82 & 115.064 & Proline & 0.019 & -0.282 \\
\hline
\end{tabular}

* 2 days and 3 days is the logarithm of the ratio of the value of value (base 2), "+" means 3 days increases relative to 2 days, " -" means decrease. VIP: Variable Importance in the Projection; RT: retention time.

Table 4. The main identification obtained between 2 days and 3 days in ESI- $(p<0.05)$.

\begin{tabular}{cccccc}
\hline VIP & RT (min) & Mass & Name & T-Test & Fold (3/2) $^{*}$ \\
\hline 1.289 & 4.578 & 192.0634 & Quinic acid & 0.019 & +25.997 \\
1.558 & 3.970 & 191.0619 & N-Acetyl-DL-methionine & 0.002 & +25.741 \\
1.810 & 6.100 & 448.1022 & Naringenin-4'-O- $\beta$-D-Glucuronide & 0.000 & +24.727 \\
1.830 & 5.969 & 332.0543 & Quercetagetin-methyl ether & 0.000 & +24.325 \\
1.295 & 9.730 & 356.1271 & Laurifolin & 0.018 & +23.249 \\
1.739 & 5.783 & 534.1009 & Luteolin 5-(6"-malonylglucoside) & 0.000 & +22.968 \\
1.588 & 5.234 & 535.1341 & Malvidin 3-(6-acetylglucoside) & 0.001 & +22.258 \\
1.264 & 5.144 & 358.068 & Dihydroxy-dimethoxy-methylenedioxy & 0.022 & +21.350 \\
1.200 & 7.953 & 610.0885 & Gallocatechin 7,4'-di-O-gallate & 0.032 & +21.183 \\
1.361 & 10.026 & 475.271 & PE (18:3) & 0.011 & +21.183 \\
1.779 & 5.245 & 318.0388 & Myricetin & 0.000 & +6.475 \\
1.254 & 11.404 & 532.1541 & Flavonol 3-O-D-xylosylglucoside & 0.023 & +5.586 \\
1.422 & 5.464 & 534.0963 & Cyanidin 3-O-(6-O-malonyl- $\beta$-D-glucoside) & 0.007 & +4.072 \\
1.737 & 5.877 & 218.1155 & Pantothenic Acid & 0.000 & +3.618 \\
1.757 & 7.131 & 302.0438 & Quercetin & 0.000 & +3.545 \\
1.323 & 8.530 & 286.0838 & Naringenin 5-methyl ether & 0.015 & +3.037 \\
1.606 & 4.247 & 304.0591 & Pentahydroxyflavanone & 0.001 & +2.656 \\
1.342 & 6.523 & 476.1319 & Naringenin 7-O-beta-D-glucoside 6"-acetate & 0.013 & +2.508 \\
1.399 & 5.708 & 450.1176 & Eriodictyol-O-glucoside & 0.008 & +2.388 \\
1.577 & 1.056 & 176.0325 & Ascorbic acid & 0.001 & -2.228 \\
1.395 & 13.135 & 278.2251 & Linolenic Acid & 0.009 & +1.268 \\
1.123 & 5.787 & 490.1128 & Cyanidin 3-(6-acetylgalactoside) & 0.049 & +1.194 \\
1.338 & 4.912 & 180.0438 & Caffeic Acid & 0.013 & -1.091 \\
1.312 & 0.721 & 422.0835 & Sucrose-6-phosphate & 0.016 & -0.608 \\
\hline
\end{tabular}

* 2 days and 3 days is the logarithm of the ratio of the value of value (base 2), "+" means 3 days increases relative to 2 days, " $"$ " means decrease. VIP: Variable Importance in the Projection; RT: retention time.

\subsubsection{Identification of Metabolites in 3-4 Days}

Comparing the samples of 3 days with 4 days, the main metabolite differences are shown in Tables 5 and 6. Overall, the metabolic differences between 3 days and 4 days were few. In the positive mode, dihydroxy-7, $2^{\prime}$-dimethoxyflavanone at 4 days compared with 3 days was increased by about 3.5 times. On the other hand, trihydroxy-3-methoxyflavone decreased by about 12 times. From the negative mode, kolaflavanone, eriodictyol, the derivative of naringenin, cyanidin, and myricetin were increased. In contrast, linolenic acid and phenylacetic acid at 4 days, compared with 3 days were decreased.

Table 5. The main identification obtained between 3 days and 4 days in ESI+ $(p<0.05)$.

\begin{tabular}{cccccc}
\hline VIP & RT $(\mathbf{m i n})$ & Mass & Name & T-Test & Fold (4/3) ${ }^{*}$ \\
\hline 1.383 & 7.64 & 300.064 & Trihydroxy-3-methoxyflavone & 0.010 & -3.618 \\
1.039 & 6.34 & 316.0954 & Dihydroxy-7,2'-dimethoxyflavanone & 0.000 & +1.796 \\
1.155 & 10.43 & 354.2779 & MG (18:2) & 0.037 & -1.697 \\
1.899 & 11.12 & 356.2934 & MG (18:1) & 0.036 & -1.169 \\
1.333 & 4.02 & 117.0579 & Indole & 0.010 & +0.508 \\
2.041 & 10.39 & 198.1623 & Lauroleic acid & 0.029 & +0.304 \\
\hline
\end{tabular}

* 3 days and 4 days is the logarithm of the ratio of the value of value (base 2), "+" means 4 days increases relative to 3 days, " -" means decrease. VIP: Variable Importance in the Projection; RT: retention time. 
Table 6. The main identification obtained between 3 days and 4 days in ESI- $(p<0.05)$.

\begin{tabular}{cccccc}
\hline VIP & RT (min) & Mass & Name & T-Test & Fold (4/3) \\
\hline 2.120 & 8.67 & 588.1278 & Kolaflavanone & 0.000 & +5.933 \\
2.104 & 9.73 & 356.1271 & Laurifolin & 0.000 & +3.667 \\
1.516 & 1.09 & 433.1176 & Cyanidin 3-rhamnoside & 0.034 & +3.486 \\
1.579 & 1.32 & 216.040 & 2-C-Methyl-D-erythritol 4-phosphate & 0.026 & -3.477 \\
2.215 & 4.87 & 138.0319 & Salicylic acid & 0.000 & +2.569 \\
1.871 & 5.50 & 302.0797 & Ferreirin & 0.004 & +2.537 \\
1.518 & 0.68 & 250.153 & Xanthoxin & 0.034 & -2.407 \\
2.173 & 7.52 & 316.0956 & Eriodictyol 7,3'-dimethyl ether & 0.000 & +2.239 \\
2.241 & 5.69 & 168.042 & Vanillic acid & 0.000 & +2.192 \\
1.664 & 7.95 & 610.0885 & Epigallocatechin 5,7-di-O-gallate & 0.017 & +2.162 \\
2.028 & 5.14 & 358.068 & Dihydroxy-dimethoxy-methylenedioxy & 0.001 & +1.843 \\
2.096 & 3.97 & 191.0619 & N-Acetyl-DL-methionine & 0.001 & +1.830 \\
1.554 & 4.97 & 450.1182 & Eriodictyol 6-O-glucoside & 0.029 & +1.777 \\
1.878 & 5.48 & 448.1021 & Naringenin 5-O-glucuronide & 0.004 & +1.723 \\
1.765 & 7.94 & 316.0593 & Quercetin 3-methyl ether & 0.009 & +1.717 \\
1.565 & 5.82 & 288.0642 & Eriodictyol & 0.027 & +1.613 \\
1.683 & 8.53 & 286.0838 & Naringenin 5-methyl ether & 0.015 & +1.450 \\
1.621 & 6.60 & 136.0522 & Phenylacetic acid & 0.021 & -1.387 \\
2.032 & 5.25 & 318.0388 & Myricetin & 0.001 & +1.256 \\
1.784 & 4.58 & 192.0634 & Quinic acid & 0.008 & +1.190 \\
1.640 & 13.13 & 278.2251 & Linolenic Acid & 0.019 & -1.021 \\
\hline
\end{tabular}

* 3 days and 4 days is the logarithm of the ratio of the value of value (base 2), "+" means 4 days increases relative to 3 days, "-" means decrease. VIP: Variable Importance in the Projection; RT: retention time.

\subsubsection{Identification of Metabolites in 4-5 Days}

Comparing the samples of 4 days with 5 days, the main metabolite differences are shown in Tables 7 and 8 . In the positive mode, dehydrophytosphingosine at 5 days, compared with 4 days, increased by about 5400 times. From the negative mode, naringenin- $4^{\prime}-O-\beta-D-$ glucuronide changes significantly. At 5 days, compared with the 4 days, it fell by about 110 times; other metabolic changes were small.

Table 7. The main identification obtained between 4 days and 5 days in ESI+ $(p<0.05)$.

\begin{tabular}{cccccc}
\hline VIP & RT (min) & Mass & Name & T-Test & Fold (5/4) * \\
\hline 1.222 & 11.44 & 315.278 & Dehydrophytosphingosine & 0.023 & +22.358 \\
\hline 1.547 & 13.14 & 278.2252 & Linolenic Acid & 0.000 & +2.291 \\
\hline 1.106 & 10.43 & 354.2779 & MG(18:2) & 0.017 & +1.874 \\
\hline 1.337 & 6.98 & 708.189 & Patuletin 3,7-(3-acetylrhamnoside) & 0.004 & +1.359 \\
\hline 1.110 & 7.64 & 300.064 & Trihydroxy-3-methoxyflavone & 0.032 & +1.167 \\
\hline 1.317 & 0.66 & 155.0697 & Histidine & 0.003 & -0.467 \\
\hline
\end{tabular}

* 4 days and 5 days is the logarithm of the ratio of the value of value (base 2), "+" means 5 days increases relative to 4 days, "-" means decrease. VIP: Variable Importance in the Projection; RT: retention time.

Table 8. The main identification obtained between 4 days and 5 days in ESI- $(p<0.05)$.

\begin{tabular}{cccccc}
\hline VIP & RT (min) & Mass & Name & T-Test & Fold (5/4) * \\
\hline 1.442 & 6.18 & 448.1026 & Naringenin-4'-O- $\beta$-D-Glucuronide & 0.015 & -23.434 \\
1.259 & 1.06 & 176.0325 & Ascorbic acid & 0.042 & -3.930 \\
1.828 & 4.39 & 130.063 & Ketoleucine & 0.000 & +2.189 \\
1.840 & 13.13 & 278.2251 & Linolenic Acid & 0.000 & +2.116 \\
1.640 & 7.07 & 264.1366 & Abscisic Acid & 0.003 & +2.097 \\
1.954 & 5.23 & 535.1341 & Malvidin 3-(6-acetylglucoside) & 0.000 & +2.016 \\
1.503 & 5.21 & 670.1768 & Limocitrin 3,7-diglucoside & 0.010 & +1.913 \\
1.515 & 4.78 & 122.0368 & Salicylaldehyde & 0.009 & -1.547 \\
1.436 & 13.73 & 280.2405 & Linoleic acid & 0.016 & 1.395 \\
\hline
\end{tabular}


Table 8. Cont.

\begin{tabular}{cccccc}
\hline VIP & RT (min) & Mass & Name & T-Test & Fold (5/4) * \\
\hline 1.694 & 1.34 & 131.0952 & Leucine & 0.002 & -1.374 \\
1.580 & 7.90 & 302.0799 & Ferreirin & 0.005 & +1.275 \\
1.238 & 3.96 & 354.0963 & Chlorogenic Acid & 0.046 & -1.241 \\
1.228 & 6.74 & 270.0534 & Trihydroxyflavone & 0.048 & -1.213 \\
1.843 & 5.69 & 168.042 & Vanillic acid & 0.000 & +1.072 \\
1.807 & 0.69 & 155.0699 & Histidine & 0.000 & -0.979 \\
1.951 & 2.22 & 148.0526 & Cinnamic acid & 0.000 & -0.827 \\
1.280 & 9.73 & 356.1271 & Laurifolin & 0.038 & +0.821 \\
1.476 & 4.58 & 376.0789 & Tetrahydroxy-3,6,2'-trimethoxyflavone & 0.012 & -0.815 \\
1.259 & 8.67 & 588.1278 & Kolaflavanone & 0.042 & +0.763 \\
1.352 & 5.89 & 194.0581 & ferulic acid & 0.026 & -0.639 \\
1.543 & 4.01 & 117.0581 & Indole & 0.007 & -0.609 \\
1.493 & 0.74 & 147.0545 & Glutamate & 0.011 & -0.503 \\
1.610 & 4.58 & 422.0842 & Sucrose-6-phosphate & 0.004 & -0.513 \\
\hline
\end{tabular}

* 4 days and 5 days is the logarithm of the ratio of the value of value (base 2$),$ " + " means 5 days increases relative to 4 days, " -" means decrease. VIP: Variable Importance in the Projection; RT: retention time.

\subsection{Biological Pathway and Function Analysis}

Using pattern recognition analysis on the metabolites, we were able to achieve a clear separation between each pair of time points. To identify the pathways involved in fresh-cut CWC etiolation, previously identified metabolites were analyzed using MetPA (Metabolomic Pathway Analysis) software. MetPA is a web-based tool that derives much of its power from the KEGG metabolic pathways database. In metabolic networks, changes in more "important" positions result in a more substantial effects on the pathway compared to changes occurring in marginal or relatively isolated positions. In our analysis, we identified several pathways that appear to be involved in etiolation, including the glycolysis pathway, fatty acid metabolic pathway, citrate cycle pathway, and flavonoid biosynthesis pathway. The green parts in Figure 4 represent the differences we have detected. In the glycolysis pathway, the $\alpha$-D-glucose is converted to $\alpha$-D-glucose- $6 \mathrm{p}$ and then further transformed into glyceraldehyde-3P, while the $\alpha$-D-glucose- $6 \mathrm{p}$ is converted to histidine; glyceraldehyde-3P and glycerate-1,3P2 are convert to each other, and at the same time converted to 2-C-methyl-D-4-erythritol-4P. Glycerate-3P and phosphoenol-pyruvate are convert to each other and generate phosphoserine. Pyruvate is created from phosphoenol-pyruvate, and converts to octanoate (8:0), triggered the citrate cycle pathway. Furthermore, pyruvic, leucine, and L-alanine interactions in the flavonoid biosynthesis pathway have a certain effect.

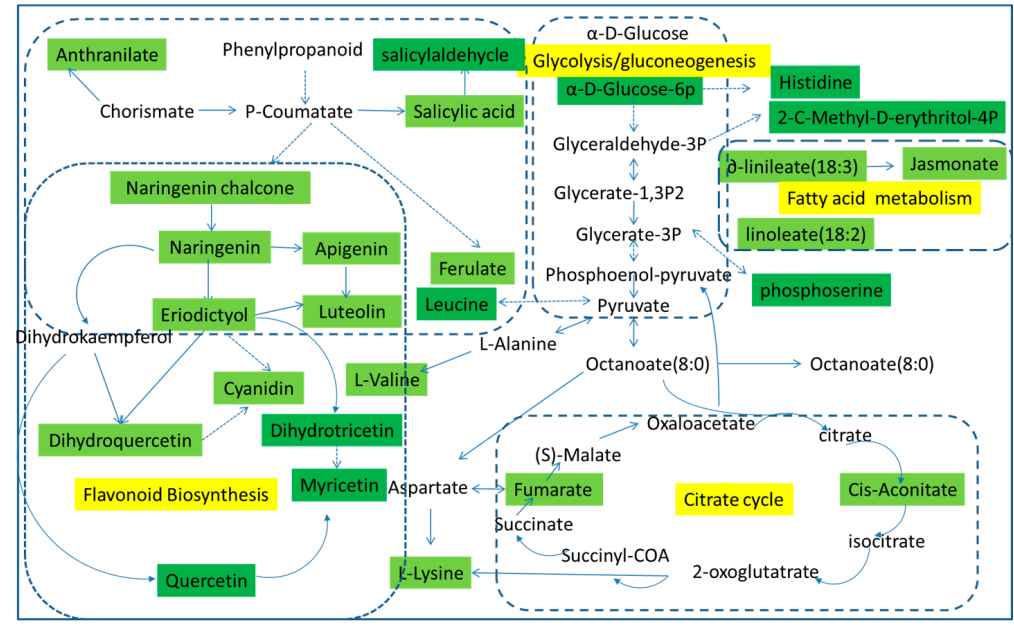

Figure 4. Metabolomic Pathway Analysis (MetPA) construction of the metabolic pathways involved in the etiolation of fresh-cut CWC. 
The major substance that causes the fresh-cut CWC yellowing are naringenin and eriodictyol, both found in the flavonoid biosynthesis pathway. Therefore, the flavonoid biosynthesis pathway is the most important pathway for CWC yellowing. As we can see from Figure 5, phenylalanine is converted to p-coumaroyl-CoA, followed by conversion to naringenin chalcone, to naringenin, and then naringenin enters different pathways. Firstly, it can transform into apigenin and its derivatives; secondly, it can produce eriodictyol and its derivatives; and thirdly it can produce dihydrokaempferol, quercetin, and myricetin. From here, eriodictyol can be further transformed to luteolin, cyanidin, dihydroquercetin, dihydrotricetin, and others. According to the metabolism pathway, quercetin, myricetin, apigenin, luteolin, and other substances were also yellowing substances. Therefore, the yellowing of fresh-cut CWC is caused by several yellow substances, and our study indicated that the flavonoid biosynthetic pathway is the main mechanism for fresh-cut CWC yellowing. Detailed construction of the yellowing metabolism pathway is shown in Figure 5.

\section{Materials and Methods}

\subsection{Chemicals, Reagents and Materials}

Acetonitrile (LC/MS grade) and methanol (HPLC grade) were purchased from Merck (Damstadt, Gemany). Formic acid was purchased from CNW (Düsseldorf, Gemany). Leucine-enkephalin was obtained from Sigma Company (San Francisco, CA, USA). Distilled water was purchased from Watsons (Hong Kong, China). NaClO were purchased from "Xiya" Company (Chengdu, China).

Fresh CWCs were purchased in Haikou, China and stored in the laboratory at $17{ }^{\circ} \mathrm{C}$. Fruits without physical damage or diseases and with uniform size were selected for analysis. CWCs were treated as reported by You [34] and Peng [35]. Fresh CWCs (100 kg) were washed, peeled using a sharp, stainless steel knife, and chopped into small, thick slices. The slices were then disinfected with $0.1 \% \mathrm{NaClO}$ for $15 \mathrm{~min}$ before airing. The CWCs were packed with plastic packaging machines and preserved at $17^{\circ} \mathrm{C}$ for 5 days.

To assess the primary metabolites as the CWCs turn yellow, CWCs were randomly selected from five different yellowing stages (Figure 5): one hour post-cutting (0 day), two days post-cutting (2 days), three days post-cutting (3 days), four days post-cutting (4 days), and five days post-cutting (5 days). Slices taken from the yellowed surface were then used as samples. Since the sample between 0 day and $1 \mathrm{~d}$ showed little difference, the sample of $1 \mathrm{~d}$ was removed from further analyses. Six samples from each of the five time stages ( $0.5 \mathrm{~g}$ each) were transferred to a $1.5 \mathrm{~mL}$ centrifuge tube, mixed with $1 \mathrm{~mL}$ solvent $\left(\mathrm{MeOH} / \mathrm{H}_{2} \mathrm{O}, 7: 3 \mathrm{v} / \mathrm{v}\right)$ by smashing for $1 \mathrm{~min}$, extracted by ultrasonication for $10 \mathrm{~min}$, and then separated by centrifugation at $12,000 \mathrm{rpm}$ at $4{ }^{\circ} \mathrm{C}$ for $15 \mathrm{~min}$. Finally, $100 \mu \mathrm{L}$ aliquots of the supernatant were transferred to autosampler vials.

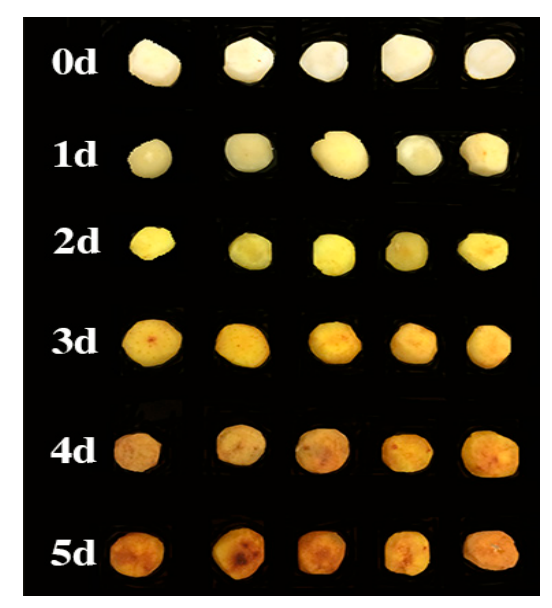

Figure 5. The yellowing process from the fresh-cut Chinese water chestnut. Each day has 5 replicate samples. 


\subsection{Metabolic Profiling and Data Analysis}

Chromatographic analysis was performed on an Agilent C18 column (Agilent, $100 \mathrm{~mm} \times 2.1 \mathrm{~mm}$, $1.8 \mu \mathrm{m})$ using an Agilent LC system (Agilent, 1290 Infinity LC, 6530 UHD, Palo Alto, CA, USA). The column temperature was maintained at $40{ }^{\circ} \mathrm{C}$ and eluted at a flow rate of $0.4 \mathrm{~mL} / \mathrm{min}$. The mobile phase consisted of a linear gradient system of (A) $0.1 \%$ formic acid in water and (B) $0.1 \%$ formic acid in acetonitrile. The gradient elution process: $0-3 \mathrm{~min}, 20 \%-50 \% \mathrm{~B} ; 3-13 \mathrm{~min}, 50 \%-95 \% \mathrm{~B} ; 13-15 \mathrm{~min}$, $95 \% \mathrm{~B}$. The injection volume was $4 \mu \mathrm{L}$ and the autosampler temperature was $4{ }^{\circ} \mathrm{C}$.

The eluent was introduced into the mass spectrometer (Agilent 6530 Accurate-Mass Q-TOF/MS). The MS system was operated using positive-ion (ESI+) and negative-ion (ESI-) modes. Conditions of positive-ion analysis were as follows: nitrogen was used as the nebulizing gas; the cone gas flow was set at $50 \mathrm{~L} / \mathrm{h}$, and the desolvation gas flow was set at $600 \mathrm{~L} / \mathrm{h}$. The capillary voltage was $4.0 \mathrm{kV}$, the sampling cone voltage was $35.0 \mathrm{kV}$, the source temperature was $100{ }^{\circ} \mathrm{C}$, the desolvation temperature was $350^{\circ} \mathrm{C}$, and the extraction cone voltage was $4.0 \mathrm{~V}$. In negative-ion mode, the cone gas flow was set at $50 \mathrm{~L} / \mathrm{h}$ and the desolvation gas flow was set at $700 \mathrm{~L} / \mathrm{h}$. The capillary voltage was $3.5 \mathrm{kV}$, the sampling cone voltage was $50 \mathrm{kV}$, the source temperature was $100{ }^{\circ} \mathrm{C}$, the desolvation temperature was $300{ }^{\circ} \mathrm{C}$, and the extraction cone voltage was $4.0 \mathrm{~V}$. The scan time was set to $0.03 \mathrm{~s}$ with a $0.02 \mathrm{~s}$ interscan time. Data were collected from 50 to $1000 \mathrm{~m} / \mathrm{z}$. For accurate and repeatable mass acquisition, a lock-mass of leucine-enkephalin was used via a lock spray interface monitor for positive-ion mode $\left([\mathrm{M}+\mathrm{H}]^{+}=556.2771 \mathrm{Da}\right)$ and negative-ion mode $\left([\mathrm{M}-\mathrm{H}]^{-}=554.2615 \mathrm{Da}\right)$ to ensure accuracy during the MS analysis.

All data were processed using Mass Profiler in the MassHunter software platform (Agilent) for LC/MS data preprocessing, followed by manual postediting in Microsoft Excel. We removed impurity peaks due to column bleeding and sample preparation. The intensity of each ion was normalized with respect to the total ion count to generate a data matrix that consisted of the rt_mass (retention time_mass), the quantity of observe, and the normalized peak. After that, the multivariate data matrix was introduced into SIMCA-P 13.0 software (Umetrics, Umeå, Sweden) for PCA. PCA data were visualized by plotting the PC scores, with each point representing one rt_mass pair. Thus, the loading plot gives an indication of the metabolites that most strongly influence the patterns in the score plot. In order to further discriminate the metabolites and biomarkers, the data were analyzed using PLS-DA and OPLS-DA methods. Variables that significantly discriminated between groups were considered potential biomarkers and were assigned with $p$-values using a student's test ( $t$-test); $p$-values less than 0.05 were considered statistically significant [36]. The identities of specific metabolites were confirmed by comparison of their mass spectra and mass-to-charge ratio $(\mathrm{m} / \mathrm{z})$ with free online databases, including METLIN (http://metlin.scripps.edu/).

The construction, interaction, and pathway analyses of potential biomarkers were performed with MetaboAnalyst 2.0, with database sources, including METLIN and KEGG (http:/ / www.genome. $\mathrm{jp} / \mathrm{kegg} /$ ), to help identify pathways that were most significantly altered.

\section{Discussion}

The yellowing of fresh-cut CWC is one of the leading causes of reduced shelf life and commercial value, and it is known to be enzymatically different from the browning of other vegetables and fruits [37-39]. Despite attempts to reduce CWC yellowing [37,40], we still lack methods to fundamentally inhibit etiolation, and the mechanism of yellowing remains unclear. The advent of the metabolomics field represents a paradigm shift in metabolic research, away from methods that focus on a limited number of single pathways and towards approaches that capture the entire complexity of metabolic networks [41]. Emerging techniques in metabolomics provide a powerful platform for discovering novel biomarkers and biochemical pathways [42]. This may allow us to develop techniques that inhibit CWC yellowing, potentially leading to future advances in inhibition of these pathways.

In this study, samples from different time points of CWC yellowing were analyzed by LC-Q/TOF-MS, coupled with multivariate data analyses; the major metabolic differences were obtained. All samples 
were successfully modeled, and we identified significant differences in PCA scores between each pair of time points. The pattern was verified as authentic by the PLS-DA analysis. Then, the difference between each pair of time points was analyzed by OPLS-DA combined with $t$-test, and the metabolites were obtained. It was found that metabolic differences include organic acids (such as salicylic acid, citric acid, ferulic acid, leucine, L-aspartic acid, lysine etc.) and flavonoids (like eriodictyol, naringenin, quercetin, myricetin, apigenin, luteolin etc.), while sucrose mainly existed in negative mode. Among those mentioned, two of the flavonoids were yellowing substances. The content changes of eriodictyol and naringenin is outstanding, which showed an upward trend that was proportional to the degree of fresh-cut CWC yellowing during the storage time. The change of myricetin showed a moderate upward trend during storage. In comparison with eriodictyol and naringenin, the change in apigenin and luteolin showed less of an upward trend and did not rise after that time, and the content of quercetin showed fluctuations in growth, which is currently under further investigation. It was demonstrated that the yellowing phenomenon of fresh-cut CWC was caused by a series of yellowing substances, mainly eriodictyol and naringenin, which is consistent with the study of Pan [21]; the content of flavonoids that cause yellowing was proportional to the degree of fresh-cut CWC yellowing. Nowadays, flavonoids are known to be key antioxidant substances that have antiaging, disease resistance, antimicrobial, anti-inflammatory, and antitumor activities, and are becoming more and more abundant in modern life. This study aims to lay the foundation for the further study of fresh-cut CWC yellowing by investigating the substances causing the phenomenon [43]. It was proved that yellowing substances of fresh-cut CWC were connected with the glycolysis pathway, fatty acid metabolism pathway, citrate cycle pathway, and flavonoid biosynthetic pathway by identifying metabolic differences combined with METLIN and KEGG data. Among the metabolites identified, organic acids began to accumulate in the initial stage of the fresh-cut CWC yellowing and were consumed as respiratory matrixes of the glycolysis pathway and citrate cycle pathway in their last stages. Organic acids also participate in respiration and metabolic processes of phenols, amino acids, esters, and flavonoids [44], explaining that organic acids increased gradually at the early time points and descend gradually at the end.

The flavonoids causing the yellowing of fresh-cut CWC mainly derived from the flavonoid biosynthetic pathway. It was proved that phenylalanine was converted into $p$-coumaric-CoA in the first step, which consistent with the study of Burbulis [45]. Naringenin, which is a significant checkpoint in the flavonoid biosynthetic pathway, is converted into the second key substance, eriodictyol, which was converted into dihydrokaempferol, then quercetin, and finally, myricetin. In the metabolic pathway, luteolin, apigenin and its derivatives, quercetin, and myricetin were also yellowing substances, in addition to naringenin and eriodictyol, which revealed that the yellowing is the result of the joint action of flavonoids, mainly naringenin and eriodictyol. This suggests that the etiolation molecules are a mixture of metabolites, but it is clear that the main substances are naringenin and eriodictyol. According to other research reports, in the tissue of grape and corn, 4-coumarin-CoA is generated by phenylalanine in the phenylpropanoid metabolic pathway, which is converted into naringenin chalcone, then naringenin, eriodictyol, and pentahydroxyflavanone were generated in the end [46]; these reports are consistent with this study. The pathway of fresh-cut CWC yellowing is complex, and each pathway contains multiple metabolites; there are rare reports about the relationship between the intermediate products and their interaction mechanism that leads to yellow that it is a complex pathway. In the further work, an in vitro model will be set up to simulate the process of flesh-cut CWC yellowing to confirm the reliability of the metabolic pathways.

\section{Conclusions}

To the best of our knowledge, this is the first report of a metabolomics study of the etiolation of fresh-cut CWC. A rapid, reliable, and sensitive LC-Q/TOF-MS method was validated for qualitative analysis of the different stages of CWC yellowing. Metabolites from each time point could be distinguished from each other by this method, which demonstrates that a significant metabolic 
perturbation is occurring. These 3008 peaks (1046 peaks in positive mode and 1962 in negative mode) could be useful as biomarkers for the different stages of yellowing. Interestingly, the flavanoid metabolic pathway emerged as a key pathway in the etiolation of fresh-cut CWC. This study also identified eriodictyol, naringenin, and sucrose as important features in this process, verifying the results of our previous study [21]. This investigation is a good starting point for providing a better theoretical understanding of how to efficiently inhibit the yellowing of CWC. However, further studies are needed for a deeper understanding of the metabolic pathways and enzymes that are associated with CWC yellowing.

Acknowledgments: This project was supported by the National Natural Science Foundation of China (Grant No. 31360414). We sincerely thank Shanghai Sensichip Infotech Co. Ltd. for their technical support.

Author Contributions: Author Yi-Xiao Li and Yong-Gui Pan designed the research and discussed the data; Yi-Xiao Li, Feng-Ping He, Shang-Bin Li and Meng-Qi Yuan performed the experiments; Yong-Gui Pan provided the sample; Yi-Xiao Li and Yong-Gui Pan analyzed the data; Yi-Xiao Li wrote the paper; Yi-Xiao Li and Yong-Gui Pan modified the language of the paper. All authors read and approved the final manuscript.

Conflicts of Interest: The authors declare no conflict of interest.

\section{References}

1. Armitage, E.G.; Barbas, C. Metabolomics in cancer biomarker discovery: Current trends and future perspectives. J. Pharm. Biomed. Anal. 2014, 87, 1-11. [CrossRef] [PubMed]

2. Li, P.L.; Sun, H.G.; Hua, Y.L.; Ji, P.; Zhang, L.; Li, J.X.; Wei, Y. Metabolomics study of hematopoietic function of Angelica sinensis on blood deficiency mice model. J. Ethnopharmacol. 2015, 166, 261-269. [CrossRef] [PubMed]

3. Zhao, Y.Y.; Zhang, L.; Long, F.Y.; Cheng, X.L.; Bai, X.; Wei, F.; Lin, R.C. UPLC-Q-TOF/HSMS/MSE-based metabonomics for adenine-induced changes in metabolic profiles of rat faeces and intervention effects of ergosta-4,6,8(14),22-tetraen-3-one. Chem. Biol. Interact. 2013, 201, 31-38. [CrossRef] [PubMed]

4. Gao, X.; Guo, M.; Zhao, B.; Peng, L.; Su, J.; Bai, X.; Li, J.; Qiao, Y. A urinary metabonomics study on biochemical changes in yeast-induced pyrexia rats: A new approach to elucidating the biochemical basis of the febrile response. Chem. Biol. Interact. 2013, 204, 39-48. [CrossRef] [PubMed]

5. Zhang, A.; Sun, H.; Han, Y.; Yuan, Y.; Wang, P.; Song, G.; Yuan, X.; Zhang, M.; Xie, N.; Wang, X. Exploratory urinary metabolic biomarkers and pathways using UPLC-Q-TOF-HDMS coupled with pattern recognition approach. Analyst 2012, 137, 4200-4208. [CrossRef] [PubMed]

6. Holmes, E.; Loo, R.L.; Stamler, J.; Bictash, M.; Yap, I.K.; Chan, Q.; Ebbels, T.; De Iorio, M.; Brown, I.J.; Veselkov, K.A. Human metabolic phenotype diversity and its association with diet and blood pressure. Nature 2008, 453, 396-400. [CrossRef] [PubMed]

7. Wang, F.; Liu, X.; Liu, C.; Liu, Z.; Sun, L. Effects of antibiotic antitumor drugs on nucleotide levels in cultured tumor cells: an exploratory method to distinguish the mechanisms of antitumor drug action based on targeted metabolomics. Acta Pharm. Sin. B 2015, 5, 223-230. [CrossRef] [PubMed]

8. Mc Auley, M.T.; Choi, H.; Mooney, K.; Paul, E.; Miller, V.M. Systems biology and synthetic biology: A new epoch for toxicology research. Adv. Toxicol. 2015, 2015, 575403. [CrossRef]

9. Freemark, M. Metabolomics in nutrition research: Biomarkers predicting mortality in children with severe acute malnutrition. Food Nutr. Bull. 2015, 36 (Suppl. S1), 88S-92S. [CrossRef]

10. Masclaux-Daubresse, C.; Clément, G.; Anne, P.; Routaboul, J.-M.; Guiboileau, A.; Soulay, F.; Shirasu, K.; Yoshimoto, K. Stitching together the multiple dimensions of autophagy using metabolomics and transcriptomics reveals impacts on metabolism, development, and plant responses to the environment in Arabidopsis. Plant Cell Online 2014, 26, 1857-1877. [CrossRef] [PubMed]

11. Wang, X.; Sun, H.; Zhang, A.; Sun, W.; Wang, P.; Wang, Z. Potential role of metabolomics apporoaches in the area of traditional Chinese medicine: As pillars of the bridge between Chinese and Western medicine. J. Pharm. Biomed. Anal. 2011, 55, 859-868. [CrossRef] [PubMed]

12. Zhang, A.; Sun, H.; Yan, G.; Wang, P.; Wang, X. Mass spectrometry-based metabolomics: Applications to biomarker and metabolic pathway research. Biomed. Chromatogr. 2016, 30, 7-12. [CrossRef] [PubMed] 
13. Chin, E.; Slupsky, C.; Weimer, B.; Slupsky, C. Applications of metabolomics in food science: Food composition and quality, sensory and nutritional attributes. In Metabolomics in Food and Nutrition; Woodhead Publ. Ltd.: Cambridge, UK, 2013; pp. 217-230.

14. McGhie, T.K.; Rowan, D.D. Metabolomics for measuring phytochemicals, and assessing human and animal responses to phytochemicals, in food science. Mol. Nutr. Food Res. 2012, 56, 147-158. [CrossRef] [PubMed]

15. Cevallos-Cevallos, J.M.; Reyes-De-Corcuera, J. Metabolomics in food science. Adv. Food Nutr. Res. 2011, 67, 1-24.

16. Cai, Y.; Gao, Y.; Tan, G.; Wu, S.; Dong, X.; Lou, Z.; Zhu, Z.; Chai, Y. Myocardial lipidomics profiling delineate the toxicity of traditional Chinese medicine Aconiti Lateralis radix praeparata. J. Ethnopharmacol. 2013, 147, 349-356. [CrossRef] [PubMed]

17. Gullberg, J.; Jonsson, P.; Nordström, A.; Sjöström, M.; Moritz, T. Design of experiments: an efficient strategy to identify factors influencing extraction and derivatization of Arabidopsis thaliana samples in metabolomic studies with gas chromatography/mass spectrometry. Anal. Biochem. 2004, 331, 283-295. [CrossRef] [PubMed]

18. De Vos, R.C.; Moco, S.; Lommen, A.; Keurentjes, J.J.; Bino, R.J.; Hall, R.D. Untargeted large-scale plant metabolomics using liquid chromatography coupled to mass spectrometry. Nat. Protoc. 2007, 2, 778-791. [CrossRef] [PubMed]

19. T'Kindt, R.; Morreel, K.; Deforce, D.; Boerjan, W.; van Bocxlaer, J. Joint GC-MS and LC-MS platforms for comprehensive plant metabolomics: Repeatability and sample pre-treatment. J. Chromatogr. B 2009, 877, 3572-3580. [CrossRef] [PubMed]

20. Yin, P.; Xu, G. Current state-of-the-art of nontargeted metabolomics based on liquid chromatography-mass spectrometry with special emphasis in clinical applications. J. Chromatogr. A 2014, 1374, 1-13. [CrossRef] [PubMed]

21. Pan, Y.-G.; Li, Y.-X.; Yuan, M.-Q. Isolation, purification and identification of etiolation substrate from fresh-cut Chinese water-chestnut (Eleocharis tuberosa). Food Chem. 2015, 186, 119-122. [CrossRef] [PubMed]

22. Pan, Y.; Chen, W. Study on the enzyme activity correlated to browning in the fresh-cut Chinese water-chestnut. Anhui Agric. Sci. 2007, 35, 9856-9857.

23. Reuben, S.; Rai, A.; Pillai, B.V.; Rodrigues, A.; Swarup, S. A bacterial quercetin oxidoreductase QuoA-mediated perturbation in the phenylpropanoid metabolic network increases lignification with a concomitant decrease in phenolamides in Arabidopsis. J. Exp. Bot. 2013, 64, 5183-5194. [CrossRef] [PubMed]

24. Yin, R.; Messner, B.; Faus-Kessler, T.; Hoffmann, T.; Schwab, W.; Hajirezaei, M.-R.; von Saint Paul, V.; Heller, W.; Schäffner, A.R. Feedback inhibition of the general phenylpropanoid and flavonol biosynthetic pathways upon a compromised flavonol-3-O-glycosylation. J. Exp. Bot. 2012, 63, 2465-2478. [CrossRef] [PubMed]

25. Lin, Y.; Jain, R.; Yan, Y. Microbial production of antioxidant food ingredients via metabolic engineering. Curr. Opin. Biotechnol. 2014, 26, 71-78. [CrossRef] [PubMed]

26. Yuan, Y.; Song, L.; Li, M.; Liu, G.; Chu, Y.; Ma, L.; Zhou, Y.; Wang, X.; Gao, W.; Qin, S. Genetic variation and metabolic pathway intricacy govern the active compound content and quality of the Chinese medicinal plant Lonicera japonica thunb. BMC Genom. 2012, 13, 195. [CrossRef] [PubMed]

27. Samanta, A.; Das, G.; Das, S.K. Roles of flavonoids in plants. Int. J. Pharm. Sci. Technol. 2011, 6, $12-35$.

28. Ibrahim Alzand, K.; Abdalkarim Mohamed, M. Flavonoids: Chemistry, Biochemistry and Antioxidant activity. J. Pharm. Res. 2012, 5, 4013.

29. Song, H.-H.; Ryu, H.W.; Lee, K.J.; Jeong, I.Y.; Kim, D.S.; Oh, S.-R. Metabolomics investigation of flavonoid synthesis in soybean leaves depending on the growth stage. Metabolomics 2014, 10, 833-841. [CrossRef]

30. Carvalho, E.; Franceschi, P.; Feller, A.; Palmieri, L.; Wehrens, R.; Martens, S. A targeted metabolomics approach to understand differences in flavonoid biosynthesis in red and yellow raspberries. Plant Physiol. Biochem. 2013, 72, 79-86. [CrossRef] [PubMed]

31. Farag, M.A.; Mohsen, M.; Heinke, R.; Wessjohann, L.A. Metabolomic fingerprints of 21 date palm fruit varieties from Egypt using UPLC/PDA/ESI-qTOF-MS and GC-MS analyzed by chemometrics. Food Res. Int. 2014, 64, 218-226. [CrossRef]

32. Yuan, S.; Yao, S.; Geng, Y.; Cai, P.; Zhang, W. Identification of Anthocyanins and Flavonols in Extract of Blueberry by Using HPLC-ESI-MS/MS. Acta Chem. Scand. 2009, 67, 318-322. 
33. Li, J.; Yang, L.; Li, Y.; Tian, Y.; Li, S.; Jiang, S.; Wang, Y.; Li, X. Metabolomics study on model rats of chronic obstructive pulmonary disease treated with Bu-Fei Jian-Pi. Mol. Med. Rep. 2015, 11, 1324-1333. [CrossRef] [PubMed]

34. You, Y.; Jiang, Y.; Sun, J.; Liu, H.; Song, L.; Duan, X. Effects of short-term anoxia treatment on browning of fresh-cut Chinese water chestnut in relation to antioxidant activity. Food Chem. 2012, 132, 1191-1196. [CrossRef]

35. Pen, L.; Jiang, Y. Effects of chitosan coating on shelf life and quality of fresh-cut Chinese water chestnut. LWT Food Sci. Technol. 2003, 36, 359-364. [CrossRef]

36. Zaykin, D.V.; Zhivotovsky, L.A.; Westfall, P.H.; Weir, B.S. Truncated product method for combining $p$-values. Genet. Epidemiol. 2002, 22, 170-185. [CrossRef] [PubMed]

37. Zvitov-Ya'ari, R.; Nussinovitch, A. Browning prevention in rehydrated freeze-dried non-blanched potato slices by electrical treatment. LWT Food Sci. Technol. 2014, 56, 194-199. [CrossRef]

38. Gomes, M.H.; Vieira, T.; Fundo, J.F.; Almeida, D.P. Polyphenoloxidase activity and browning in fresh-cut 'Rocha'pear as affected by $\mathrm{pH}$, phenolic substrates, and antibrowning additives. Postharvest Biol. Technol. 2014, 91, 32-38. [CrossRef]

39. Sagar, V.; Kumar, P.S. Recent advances in drying and dehydration of fruits and vegetables: A review. J. Food Sci. Technol. 2010, 47, 15-26. [CrossRef] [PubMed]

40. Zhou, D.; Li, L.; Wu, Y.; Fan, J.; Ouyang, J. Salicylic acid inhibits enzymatic browning of fresh-cut Chinese chestnut (Castanea mollissima) by competitively inhibiting polyphenol oxidase. Food Chem. 2015, 171, 19-25. [CrossRef] [PubMed]

41. Pah, A.; Guimera, R.; Mustoe, A.; Amaral, L. Use of a global metabolic network to curate organismal metabolic networks. Sci. Rep. 2013, 3, 1695. [CrossRef] [PubMed]

42. Bai, Y.; Zhang, H.; Sun, X.; Sun, C.; Ren, L. Biomarker identification and pathway analysis by serum metabolomics of childhood acute lymphoblastic leukemia. Clin. Chim. Acta 2014, 436, 207-216. [CrossRef] [PubMed]

43. Pereira, D.M.; Valentão, P.; Pereira, J.A.; Andrade, P.B. Phenolics: From chemistry to biology. Molecules 2009, 14, 2202-2211. [CrossRef]

44. Zhang, J. Metabolic Profiling of the Major Fruit Quality Components during the Development of Fragaria ananassa "Fengxiang" Fruits; Anhui Agriculture University: Hefei, China, 2009.

45. Burbulis, I.E.; Winkel-Shirley, B. Interactions among enzymes of the Arabidopsis flavonoid biosynthetic pathway. Proc. Natl. Acad. Sci. USA 1999, 96, 12929-12934. [CrossRef] [PubMed]

46. Ferreyra, M.F.; Rius, S.P.; Casati, P. Flavonoids: Biosynthesis, biological functions, and biotechnological applications. Front. Plant Sci. 2012, 3, 1-15.

Sample Availability: Not Available.

(C) 2016 by the authors; licensee MDPI, Basel, Switzerland. This article is an open access article distributed under the terms and conditions of the Creative Commons Attribution (CC-BY) license (http://creativecommons.org/licenses/by/4.0/). 